(Supporting Information)

\title{
Remarkable Substituent Effects on the Oxidizing Ability of Triarylbismuth Dichlorides in Alcohol Oxidation
}

Yoshihiro Matano, * Teppei Hisanaga, Hisatsugu Yamada, Shingo Kusakabe, Hazumi Nomura, and Hiroshi Imahori

Department of Molecular Engineering, Graduate School of Engineering, Kyoto University, Nishikyo-ku, Kyoto 615-8510, Japan and Department of Chemistry, Graduate School of Science, Kyoto University, Sakyo-ku, Kyoto 606-8502, Japan

Contents

General

S2

Spectral and analytical data of $\mathbf{1 h}-\mathbf{u}$

S2

${ }^{1} \mathrm{H}$ NMR data of 2

S4 
General. All melting points are uncorrected. ${ }^{1} \mathrm{H}$ and ${ }^{13} \mathrm{C}$ NMR spectra were recorded using $\mathrm{CDCl}_{3}$ as the solvent unless otherwise noted. Chemical shifts are reported as the relative value vs. tetramethylsilane and $J$ values are given in Hz. FABMS spectra were measured using $m$-nitrobenzyl alcohol as a matrix. Column chromatography was performed on silica gel or on a fast flow liquid chromatography system fitted with a silica gel column. $\mathrm{CH}_{2} \mathrm{Cl}_{2}$ was distilled from $\mathrm{CaH}_{2}$ before use. $\mathrm{CDCl}_{3}$ was distilled from $\mathrm{CaH}_{2}$ and stored over $4 \AA$ molecular sieves. Toluene was distilled from sodium and stored over $4 \AA$ molecular sieves.

\section{Spectral and analytical data of $1 \mathrm{~h}-\mathrm{u}$.}

Tris(4-methoxy-2-methylphenyl)bismuth Dichloride (1h): $\mathrm{mp} 210-220{ }^{\circ} \mathrm{C}$ (decomp.); ${ }^{1} \mathrm{H}$ NMR $2.70(\mathrm{~s}, 9 \mathrm{H}, \mathrm{Me}), 3.85$ (s, 9H, OMe), 6.95-6.99 (m, 6H), 7.94 (d, 3H, J=8.4 Hz); FABMS m/z 607 $\left(\mathrm{M}^{+} \square \mathrm{Cl}\right)$. Anal. Calcd for $\mathrm{C}_{24} \mathrm{H}_{27} \mathrm{BiCl}_{2} \mathrm{O}_{3}: \mathrm{C}, 44.81 ; \mathrm{H}, 4.23$. Found: $\mathrm{C}, 45.04 ; \mathrm{H}, 3.93$.

Tris(2,4-dimethylphenyl)bismuth Dichloride (1i): mp $170-174{ }^{\circ} \mathrm{C}$ (decomp.) (lit. $\left.169{ }^{\circ} \mathrm{C},{ }^{5 b}\right) ;{ }^{1} \mathrm{H}$ NMR $\square 2.40$ (s, 9H, Me), 2.69 (s, 9H, Me), $7.28(\mathrm{dd}, 3 \mathrm{H}, J=8.4,2.8 \mathrm{~Hz}), 7.33(\mathrm{~d}, 3 \mathrm{H}, J=2.8 \mathrm{~Hz})$, $7.90(\mathrm{~d}, 3 \mathrm{H}, J=8.4 \mathrm{~Hz})$; FABMS $m / z .559\left(\mathrm{M}^{+} \square \mathrm{Cl}\right)$.

Tris(4-chloro-2-methylphenyl)bismuth Dichloride (1j): mp $154-156{ }^{\circ} \mathrm{C}$ (decomp.); ${ }^{1} \mathrm{H}$ NMR $\square 2.70$ (s, 9H, Me), $7.47(\mathrm{dd}, 3 \mathrm{H}, J=8.4,2.4 \mathrm{~Hz}), 7.53(\mathrm{~d}, 3 \mathrm{H}, J=2.4 \mathrm{~Hz}), 7.94(\mathrm{~d}, 3 \mathrm{H}, J=8.4 \mathrm{~Hz})$; FABMS $m / z 619\left(\mathrm{M}^{+} \square \mathrm{Cl}\right)$. Anal. Calcd for $\mathrm{C}_{21} \mathrm{H}_{18} \mathrm{BiCl}_{5}$ : C, 38.41; H, 2.76. Found: C, 38.28; H, 2.63.

Tris(2-methyl-4-nitrophenyl)bismuth Dichloride (1k): mp 159-162 ${ }^{\circ} \mathrm{C}$ (decomp.); ${ }^{1} \mathrm{H} \mathrm{NMR}\left(\mathrm{CDCl}_{3}\right)$ $\square 2.85(\mathrm{~s}, 9 \mathrm{H}, \mathrm{Me}), 8.21(\mathrm{~d}, 3 \mathrm{H}, J=8.8 \mathrm{~Hz}), 8.36(\mathrm{dd}, 3 \mathrm{H}, J=8.8,2.4 \mathrm{~Hz}), 8.45(\mathrm{~d}, 3 \mathrm{H}, J=2.4 \mathrm{~Hz})$; FABMS $m / z, 652\left(\mathrm{M}^{+} \square \mathrm{Cl}\right)$. Anal. Calcd for $\mathrm{C}_{21} \mathrm{H}_{18} \mathrm{BiCl}_{2} \mathrm{~N}_{3} \mathrm{O}_{6}: \mathrm{C}, 36.65 ; \mathrm{H}, 2.64 ; \mathrm{N}, 6.11$. Found: $\mathrm{C}$, $36.87 ; \mathrm{H}, 2.66 ; \mathrm{N}, 5.86$.

Tris(2-ethylphenyl)bismuth Dichloride (1l): mp $127-129{ }^{\circ} \mathrm{C}$ (decomp.); ${ }^{1} \mathrm{H} \mathrm{NMR}\left(\mathrm{CDCl}_{3}\right) \square 1.37$ (t, $9 \mathrm{H}, J=7.2 \mathrm{~Hz}, \mathrm{Me}), 3.03\left(\mathrm{q}, 6 \mathrm{H}, J=7.2 \mathrm{~Hz}, \mathrm{CH}_{2}\right), 7.48(\mathrm{dt}, 3 \mathrm{H}, J=2.0,7.6 \mathrm{~Hz}), 7.52(\mathrm{dt}, 3 \mathrm{H}, J=1.6$, 
7.6 Hz), $7.66(\mathrm{dd}, 3 \mathrm{H}, J=2.0,7.6 \mathrm{~Hz}), 8.01(\mathrm{dt}, 3 \mathrm{H}, J=1.6,7.6 \mathrm{~Hz})$; FABMS m/z $559\left(\mathrm{M}^{+} \square \mathrm{Cl}\right)$. Anal.

Calcd for $\mathrm{C}_{24} \mathrm{H}_{27} \mathrm{BiCl}_{2}$ : C, 48.42; H, 4.57. Found: C, 48.13; H, 4.54.

Tris[2-(trifluoromethyl)phenyl]bismuth Dichloride (1m): mp 127-129 ${ }^{\circ} \mathrm{C}$ (decomp.); ${ }^{1} \mathrm{H}$ NMR $\left(\mathrm{CDCl}_{3}\right) \square 7.74(\mathrm{t}, 3 \mathrm{H}, J=7.8 \mathrm{~Hz}), 7.84(\mathrm{dt}, 3 \mathrm{H}, J=1.6,7.8 \mathrm{~Hz}), 8.00(\mathrm{dd}, 3 \mathrm{H}, J=7.6,1.6 \mathrm{~Hz}), 8.22$

$(\mathrm{d}, 3 \mathrm{H}, J=8.0 \mathrm{~Hz})$; FABMS $m / z 679\left(\mathrm{M}^{+} \square \mathrm{Cl}\right)$. Anal. Calcd for $\mathrm{C}_{21} \mathrm{H}_{12} \mathrm{BiCl}_{2} \mathrm{~F}_{9}$ : C, 35.27; H, 1.69.

Found: C, 35.03; H, 1.89 .

(4-Methoxyphenyl)bis(4-methylphenyl)bismuth Dichloride (1n): mp $127-129{ }^{\circ} \mathrm{C}$ (lit. ${ }^{\text {5a }} 120-122{ }^{\circ} \mathrm{C}$ );

${ }^{1} \mathrm{H} \mathrm{NMR}\left(\mathrm{CDCl}_{3}\right): \square 2.41(\mathrm{~s}, 6 \mathrm{H}, \mathrm{Me}), 3.85(\mathrm{~s}, 3 \mathrm{H}, \mathrm{OMe}), 7.12(\mathrm{~d}, 2 \mathrm{H}, J=8.6 \mathrm{~Hz}), 7.44(\mathrm{~d}, 4 \mathrm{H}, J=8.2$

$\mathrm{Hz}), 8.35(\mathrm{~d}, 4 \mathrm{H}, J=8.2 \mathrm{~Hz}), 8.45(\mathrm{~d}, 2 \mathrm{H}, J=8.6 \mathrm{~Hz})$; FABMS $m / z 533\left(\mathrm{M}^{+} \square \mathrm{Cl}\right)$. Anal. Calcd for

$\mathrm{C}_{21} \mathrm{H}_{21} \mathrm{BiCl}_{2} \mathrm{O}: \mathrm{C}, 44.31 ; \mathrm{H}, 3.72$. Found: C, 44.29; H, 3.73.

Bis(4-methoxyphenyl)(phenyl)bismuth Dichloride (1o): glassy solid; ${ }^{1} \mathrm{H}$ NMR $\left(\mathrm{CDCl}_{3}\right): \square 3.85$ (s, $6 \mathrm{H}, \mathrm{OMe}), 7.13(\mathrm{~d} .4 \mathrm{H}, J=9.1 \mathrm{~Hz}), 7.53(\mathrm{~d}, 1 \mathrm{H}, J=7.2 \mathrm{~Hz}), 7.64(\mathrm{t}, 2 \mathrm{H}, J=7.2 \mathrm{~Hz}), 8.45(\mathrm{~d}, 4 \mathrm{H}, J=$ 9.1 Hz), 8.44 $\square 8.47$ (m, 2H); FABMS m/z $535\left(\mathrm{M}^{+} \square \mathrm{Cl}\right)$. A good analytical data for $\mathbf{1 0}$ was not obtained, although the spectral data indicated its high state of purity.

Bis(4-chlorophenyl)(4-methoxyphenyl)bismuth Dichloride (1p): mp 133-135 ${ }^{\circ} \mathrm{C} ;{ }^{1} \mathrm{H}$ NMR $\left(\mathrm{CDCl}_{3}\right)$ : $\square 3.86(\mathrm{~s}, 3 \mathrm{H}, \mathrm{OMe}), 7.15(\mathrm{~d}, 2 \mathrm{H}, J=9.0 \mathrm{~Hz}), 7.61(\mathrm{~d}, 4 \mathrm{H}, J=8.8 \mathrm{~Hz}), 8.43(\mathrm{~d}, 2 \mathrm{H}, J=9.0 \mathrm{~Hz}), 8.45$ (d, 4H, $J=8.8 \mathrm{~Hz})$; FABMS m/z $573\left(\mathrm{M}^{+} \square \mathrm{Cl}\right)$. Anal. Calcd for $\mathrm{C}_{19} \mathrm{H}_{15} \mathrm{BiCl}_{4} \mathrm{O}: \mathrm{C}, 37.40 ; \mathrm{H}, 2.48$. Found: C, 36.94; H, 2.09 .

Bis(4-chlorophenyl)(4-trifluoromethylphenyl)bismuth Dichloride (1q): mp $118-120^{\circ} \mathrm{C}$ (decomp.); ${ }^{1} \mathrm{H}$ NMR $\left(\mathrm{CDCl}_{3}\right): \square 7.64(\mathrm{~d}, 4 \mathrm{H}, J=6.9 \mathrm{~Hz}), 7.91(\mathrm{~d}, 2 \mathrm{H}, J=8.1 \mathrm{~Hz}), 8.49(\mathrm{~d}, 4 \mathrm{H}, J=6.9 \mathrm{~Hz}), 8.65$ $(\mathrm{d}, 2 \mathrm{H}, J=8.1 \mathrm{~Hz})$; FABMS $m / z 611\left(\mathrm{M}^{+} \square \mathrm{Cl}\right)$. Anal. Calcd for $\mathrm{C}_{19} \mathrm{H}_{12} \mathrm{BiCl}_{4} \mathrm{~F}_{3}: \mathrm{C}, 35.21 ; \mathrm{H}, 1.87$. Found: C, 34.99; H, 1.86 .

(4-Methoxy-2-methylphenyl)bis(2-methylphenyl)bismuth Dichloride (1r): mp 172-174 ${ }^{\circ} \mathrm{C}$ (decomp.); ${ }^{1} \mathrm{H}$ NMR $\left(\mathrm{CDCl}_{3}\right)$ : $\square 2.71$ (s, 3H, Me), 2.74 (s, 6H, Me), 3.86 (s, 3H, OMe), 6.97ロ7.00 (m, 
2H), 7.42־7.54 (m, 6H), $7.97(\mathrm{~d}, J=8.8 \mathrm{~Hz}, 1 \mathrm{H}), 8.02(\mathrm{dd}, J=1.6,7.6 \mathrm{~Hz}, 2 \mathrm{H}) ;$ FABMS $m / z 547$

$\left(\mathrm{M}^{+} \square \mathrm{Cl}\right)$. Anal. Calcd for $\mathrm{C}_{22} \mathrm{H}_{23} \mathrm{BiCl}_{2} \mathrm{O}: \mathrm{C}, 45.30 ; \mathrm{H}, 3.97$. Found: C, 45.19; H, 3.85.

(2,4-Dimethylphenyl)bis(2-methylphenyl)bismuth Dichloride (1s): mp $156-161{ }^{\circ} \mathrm{C} ;{ }^{1} \mathrm{H}$ NMR

$\left(\mathrm{CDCl}_{3}\right): \square 2.41(\mathrm{~s}, 3 \mathrm{H}, \mathrm{Me}), 2.70(\mathrm{~s}, 3 \mathrm{H}, \mathrm{Me}), 2.74(\mathrm{~s}, 6 \mathrm{H}, \mathrm{Me}), 7.26 \square 7.54(\mathrm{~m}, 8 \mathrm{H}), 7.92(\mathrm{~d}, J=8.0$

$\mathrm{Hz}, 1 \mathrm{H}), 8.03(\mathrm{~d}, J=7.2 \mathrm{~Hz}, 2 \mathrm{H})$; FABMS $m / z 531\left(\mathrm{M}^{+} \square \mathrm{Cl}\right)$. Anal. Calcd for $\mathrm{C}_{22} \mathrm{H}_{23} \mathrm{BiCl}_{2}: \mathrm{C}, 46.58 ; \mathrm{H}$, 4.09. Found: C, 46.11; H, 4.03.

(4-Chloro-2-methylphenyl)bis(2-methylphenyl)bismuth Dichloride (1t): mp 92-95 ${ }^{\circ} \mathrm{C} ;{ }^{1} \mathrm{H}$ NMR $\left(\mathrm{CDCl}_{3}\right): \square 2.72(\mathrm{~s}, 3 \mathrm{H}, \mathrm{Me}), 2.74(\mathrm{~s}, 6 \mathrm{H}, \mathrm{Me}), 7.44 \square 7.57(\mathrm{~m}, 8 \mathrm{H}), 7.98 \square 8.03(\mathrm{~m}, 3 \mathrm{H}) ; \mathrm{FABMS} m / z 551$ $\left(\mathrm{M}^{+} \square \mathrm{Cl}\right)$. Anal. Calcd for $\mathrm{C}_{21} \mathrm{H}_{20} \mathrm{BiCl}_{3}$ : C, 42.92; H, 3.43. Found: C, 42.63; H, 3.63.

(4-Chlorophenyl)bis(2-methylphenyl)bismuth Dichloride (1u): mp $163-165{ }^{\circ} \mathrm{C} ;{ }^{1} \mathrm{H} \mathrm{NMR}\left(\mathrm{CDCl}_{3}\right)$ : $\square 2.67(\mathrm{~s}, 6 \mathrm{H}, \mathrm{Me}), 7.40 \square 7.51(\mathrm{~m}, 6 \mathrm{H}), 7.71(\mathrm{~d}, J=8.4 \mathrm{~Hz}, 2 \mathrm{H}), 7.89(\mathrm{~d}, J=7.6 \mathrm{~Hz}, 2 \mathrm{H}), 8.81(\mathrm{~d}, J=$ $8.8 \mathrm{~Hz}, 2 \mathrm{H})$; FABMS m/z $537\left(\mathrm{M}^{+} \square \mathrm{Cl}\right)$. Anal. Calcd for $\mathrm{C}_{20} \mathrm{H}_{18} \mathrm{BiCl}_{3}: \mathrm{C}, 41.87 ; \mathrm{H}, 3.16$. Found: $\mathrm{C}$, 41.62; H, 3.24.

${ }^{1} \mathbf{H}$ NMR data of 2. The structures of 2 were characterized by ${ }^{1} \mathrm{H}$ NMR. In all compounds, the protonated DBU group ([DBUH $]^{+}$) was observed at $\square$ 1.6-3.5 (total $16 \mathrm{H}$ ) and at around $\square 10-11$ (br-s, $1 \mathrm{H}, \mathrm{NH})$. The protons due to the aryl ligands are summarized below.

2a: $\square 7.27(\mathrm{t}, 2 \mathrm{H}, J=7.4 \mathrm{~Hz}), 7.53(\mathrm{t}, 4 \mathrm{H}, J=7.4 \mathrm{~Hz}), 8.60(\mathrm{~d}, 4 \mathrm{H}, J=7.4 \mathrm{~Hz})$.

2b: $\square 3.78$ (s, 6H, OMe), 7.03 (d, 4H, $J=8.8 \mathrm{~Hz}), 8.47$ (d, 4H, $J=8.8 \mathrm{~Hz})$.

2c: $\square 2.28$ (s, 6H, Me), $7.32(\mathrm{~d}, 4 \mathrm{H}, J=7.6 \mathrm{~Hz}), 8.49(\mathrm{~d}, 4 \mathrm{H}, J=7.6 \mathrm{~Hz})$.

2d: $\square 7.45(\mathrm{~d}, 4 \mathrm{H}, J=8.4 \mathrm{~Hz}), 8.52(\mathrm{~d}, 4 \mathrm{H}, J=8.4 \mathrm{~Hz})$.

2e: $\square 7.71(\mathrm{~d}, 4 \mathrm{H}, J=7.6 \mathrm{~Hz}), 8.74(\mathrm{~d}, 4 \mathrm{H}, J=7.6 \mathrm{~Hz})$.

2f: $\square 8.25(\mathrm{~d}, 4 \mathrm{H}, J=8.6 \mathrm{~Hz}), 8.69(\mathrm{~d}, 4 \mathrm{H}, J=8.6 \mathrm{~Hz})$.

2g: $\square 2.58(\mathrm{~s}, 6 \mathrm{H}), 7.31(\mathrm{dd}, 2 \mathrm{H}, J=7.6,7.3 \mathrm{~Hz}), 7.44(\mathrm{dd}, 2 \mathrm{H}, J=7.6,7.3 \mathrm{~Hz}), 7.48(\mathrm{~d}, 2 \mathrm{H}, J=7.6$ 
$\mathrm{Hz}), 8.49(\mathrm{~d}, 2 \mathrm{H}, J=7.6 \mathrm{~Hz})$,

2h: $\square 2.47$ (s, 6H, Me), 3.79 (s, 6H, OMe), 6.95 (d, 2H, J= $7.2 \mathrm{~Hz}), 6.97$ (s, 2H), 8.27 (d, 2H, $J=7.2$ $\mathrm{Hz})$.

2i: $\square 2.30$ (s, 6H, Me), 2.48 (s, 6H, Me), 7.29 (d, 2H, J = $7.4 \mathrm{~Hz}), 7.30$ (s, 2H), 8.36 (d, 2H, J = 7.4 Hz).

2j: $\square 2.59$ (s, 6H, Me), $7.28(\mathrm{dd}, 2 \mathrm{H}, J=7.6,1.6 \mathrm{~Hz}), 7.38(\mathrm{~d}, 2 \mathrm{H}, J=1.6 \mathrm{~Hz}), 8.32(\mathrm{~d}, 2 \mathrm{H}, J=7.6 \mathrm{~Hz})$.

2k: $\square 2.47(\mathrm{~s}, 6 \mathrm{H}), 8.03(\mathrm{~d}, 2 \mathrm{H}, J=8.8 \mathrm{~Hz}), 8.18(\mathrm{~s}, 3 \mathrm{H}), 8.40(\mathrm{~d}, 3 \mathrm{H}, J=8.8 \mathrm{~Hz})$.

2l: $\square 1.24(\mathrm{t}, 6 \mathrm{H}, J=7.3 \mathrm{~Hz}), 2.65(\mathrm{q}, 4 \mathrm{H}, J=7.3 \mathrm{~Hz}), 7.35(\mathrm{~d}, 2 \mathrm{H}, J=7.2 \mathrm{~Hz}), 7.40-7.50(\mathrm{~m}, 4 \mathrm{H})$, 8.30 (br-s, 2H).

2m: $\square 7.47(\mathrm{t}, 3 \mathrm{H}, J=7.4 \mathrm{~Hz}), 7.67(\mathrm{t}, 3 \mathrm{H}, J=7.4 \mathrm{~Hz}), 7.90(\mathrm{~d}, 3 \mathrm{H}, J=7.8 \mathrm{~Hz}), 8.70(\mathrm{~d}, 3 \mathrm{H}, J=7.6$ $\mathrm{Hz})$.

2n: $\square 2.32$ (s, 3H, Me), $3.78(\mathrm{~s}, 3 \mathrm{H}, \mathrm{OMe}), 7.05$ (d, 2H, J=8.4 Hz), 7.36 (d, 2H, J= $7.6 \mathrm{~Hz}), 8.43$ (d, $2 \mathrm{H}, J=8.4 \mathrm{~Hz}), 8.45(\mathrm{~d}, 2 \mathrm{H}, J=7.6 \mathrm{~Hz})$.

2o: $\square 3.78(\mathrm{~s}, 3 \mathrm{H}, \mathrm{OMe}), 7.03(\mathrm{~d}, 2 \mathrm{H}, J=8.4 \mathrm{~Hz}), 7.25(\mathrm{t}, 2 \mathrm{H}, J=7.6 \mathrm{~Hz}), 7.51(\mathrm{t}, 1 \mathrm{H}, J=7.6 \mathrm{~Hz})$, $8.49(\mathrm{~d}, 2 \mathrm{H}, J=8.4 \mathrm{~Hz}), 8.55(\mathrm{~d}, 2 \mathrm{H}, J=7.6 \mathrm{~Hz})$.

2p: $\square 3.78(\mathrm{~s}, 3 \mathrm{H}, \mathrm{OMe}), 7.05(\mathrm{~d}, 2 \mathrm{H}, J=8.8 \mathrm{~Hz}), 7.44(\mathrm{~d}, 2 \mathrm{H}, J=8.0 \mathrm{~Hz}), 8.45(\mathrm{~d}, 2 \mathrm{H}, J=8.8 \mathrm{~Hz})$, $8.51(\mathrm{~d}, 2 \mathrm{H}, J=8.0 \mathrm{~Hz})$.

2q: $\square 7.47(\mathrm{~d}, 2 \mathrm{H}, J=8.0 \mathrm{~Hz}), 7.71(\mathrm{~d}, 2 \mathrm{H}, J=7.8 \mathrm{~Hz}), 8.57(\mathrm{~d}, 2 \mathrm{H}, J=8.0 \mathrm{~Hz}), 8.73(\mathrm{~d}, 2 \mathrm{H}, J=7.8$ $\mathrm{Hz})$.

2r: $\square 2.57$ (s, 3H, Me), $2.58(\mathrm{~s}, 3 \mathrm{H}, \mathrm{Me}), 3.78$ (s, 3H, OMe), $6.90(\mathrm{dd}, 1 \mathrm{H}, J=8.4,2.4 \mathrm{~Hz}), 6.97(\mathrm{~d}, 1 \mathrm{H}$, $J=2.4 \mathrm{~Hz}), 7.30(\mathrm{t}, 1 \mathrm{H}, J=7.6 \mathrm{~Hz}), 7.40-7.50(\mathrm{~m}, 2 \mathrm{H}), 8.33(\mathrm{~d}, 1 \mathrm{H}, J=8.4 \mathrm{~Hz}), 8.50(\mathrm{~d}, 1 \mathrm{H}, J=7.6$ $\mathrm{Hz})$.

2s: $\square 2.31(\mathrm{~s}, 3 \mathrm{H}, \mathrm{Me}), 2.55$ (s, 3H, Me), 2.57 (s, 3H, Me), 7.29 (d, 1H $\square 2, J=7.6 \mathrm{~Hz}), 7.34$ (s, 1H), $7.40-7.50(\mathrm{~m}, 2 \mathrm{H}), 8.34(\mathrm{~d}, 1 \mathrm{H}, J=7.6 \mathrm{~Hz}), 8.48(\mathrm{~d}, 1 \mathrm{H}, J=7.6 \mathrm{~Hz})$.

2t: $\square 2.57(\mathrm{~s}, 3 \mathrm{H}, \mathrm{Me}), 2.63(\mathrm{~s}, 3 \mathrm{H}, \mathrm{Me}), 7.30(\mathrm{~d}, 1 \mathrm{H}, J=7.6 \mathrm{~Hz}), 7.31(\mathrm{~d}, 1 \mathrm{H}, J=7.6 \mathrm{~Hz}), 7.40(\mathrm{~d}, 1 \mathrm{H}$, 
$J=7.6 \mathrm{~Hz}), 7.40-7.50(\mathrm{~m}, 2 \mathrm{H}), 8.39(\mathrm{~d}, 1 \mathrm{H}, J=7.6 \mathrm{~Hz}), 8.45(\mathrm{~d}, 1 \mathrm{H}, J=7.6 \mathrm{~Hz})$.

2u: $\square 2.67(\mathrm{~s}, 3 \mathrm{H}, \mathrm{Me}), 7.15-7.25(\mathrm{~m}, 1 \mathrm{H}), 7.40-7.45(\mathrm{~m}, 2 \mathrm{H}), 7.50(\mathrm{~d}, 2 \mathrm{H}, J=8.4 \mathrm{~Hz}), 8.36(\mathrm{dd}, 1 \mathrm{H}, J$ $=7.6,1.6 \mathrm{~Hz}), 8.58(\mathrm{~d}, 2 \mathrm{H}, J=8.4 \mathrm{~Hz})$. 